\title{
Some Graphical Methods for Determining Centres of Gravity.
}

\author{
By Dr R. F、 Muirhead.
}

(Read 13th March 1914. Received 31st March 1914).

\section{Polygonal Plane Areas (First Method).}

This method is associated with the well-known construction of a triangle equal in area to a given polygon.

In Fig. 1 let $A B C D D_{1} C_{1} B_{1} A$ be the polygonal circuit enclosing the area, and let $D D_{1}$ be any side not adjacent to $A$.

\begin{tabular}{|c|c|c|c|c|c|c|c|}
\hline et & $\mathrm{BC}^{\prime}$ & $\|$ & to & $\mathrm{AC}$ & meet & $\mathrm{CD}$ in & $\mathrm{C}^{\prime}$. \\
\hline , & $\mathbf{C}^{\prime} \mathbf{D}^{\prime}$ & , & " & $\mathrm{AD}$ & " & $\mathrm{DD}_{1}$ & $\mathrm{D}^{\prime}$. \\
\hline " & $\mathrm{B}_{1} \mathrm{C}_{1}{ }^{\prime}$ & ", & $"$ & $\mathrm{AC}_{1}$ & & $\mathrm{C}_{1} \mathrm{D}_{1}$ & $\mathrm{C}_{1}^{\prime}$. \\
\hline$"$ & $\mathrm{C}_{1}{ }^{\prime} \mathrm{D}_{1}{ }^{\prime}$ & , & ", & $A D_{1}$ & & $D_{1} \mathrm{D}$ & $D_{1}^{\prime}$. \\
\hline ", & $\mathrm{C} c$ & ", & $"$ & $\mathrm{BD}$ & & $\mathrm{BC}^{\prime}$ & \\
\hline & $\mathrm{D} d$ & $"$ & $"$ & $\mathrm{D}_{1}^{\prime} c$ & $"$ & $D^{\prime} c$ & $d$. \\
\hline & $\mathrm{C}_{1} \mathrm{c}_{1}$ & $"$ & " & $B_{1} D_{1}$ & " & $\mathrm{B}_{1} \mathrm{C}$ & $c_{1}$. \\
\hline & $\mathrm{D}_{1} d_{1}$ & & & $\mathrm{D}^{\prime} c_{3}$ & & $\mathrm{D}_{1}^{\prime} c_{1}$, & \\
\hline
\end{tabular}

Let $O$ be the mid point of $\mathrm{D}^{\prime} \mathrm{D}_{1}^{\prime}$, and let $\mathrm{AO}$ be produced to $\mathbf{A}^{\prime}$ so that $\mathrm{OA}^{\prime}=\mathrm{AO}$.

Complete the parallelograms $d \mathrm{D}^{\prime} \mathrm{A}^{\prime} d^{\prime}, d_{1} \mathrm{D}_{1}^{\prime} d^{\prime} d_{1}^{\prime}$.

Join $A d_{1}^{\prime}$ and from it cut off $\mathrm{AG}$ equal to $\frac{1}{3} \mathrm{~A} d_{1}^{\prime}$.

Then $G$ is the centre of gravity of the given polygonal area.

To explain the construction we note that the triangle $A D^{\prime} \mathrm{D}_{1}^{\prime}$ is equal in area to the given polygon. The rest of the construction depends on the principle that if a part of a body have its centre of gravity displaced by a step $s$, the corresponding displacement of the centre of gravity of the whole will be $\equiv$ as where $\alpha$ is the ratio of the mass of the part to the mass of the whole.

$N . B .-$ Here, and in what follows, we use the symbol $\equiv$ to indicate equality of length combined with sameness of direction and sense. 


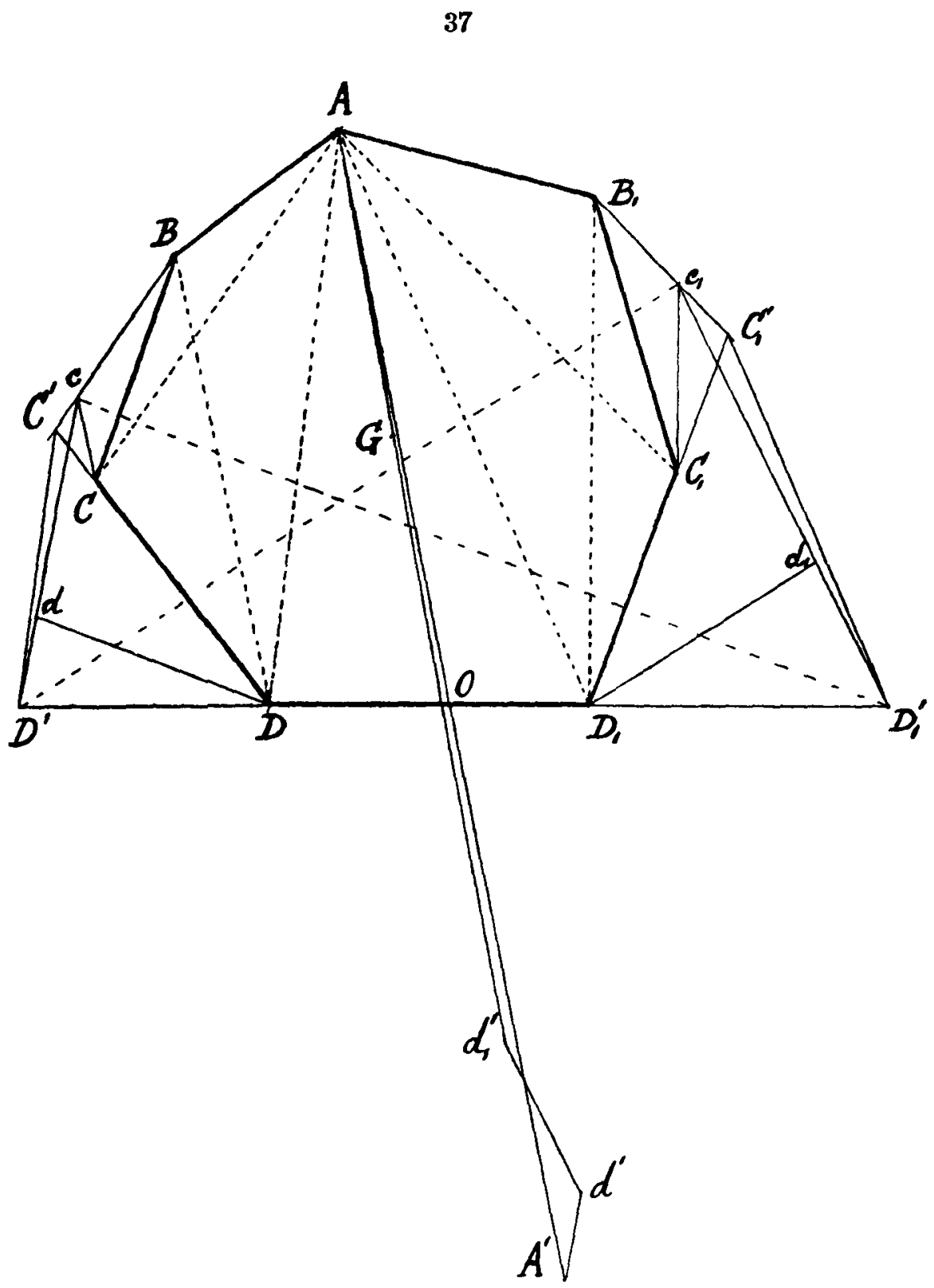

Fig. 1. 
Now if the triangular area $A B C$ is shifted to $A C^{\prime} C$, its centre of gravity is displaced through a step which is $\equiv \frac{1}{3} \mathrm{BC}^{\prime}$. It follows that the centre of gravity of the area which was originally ABCDA and becomes changed to $A C^{\prime} D A$ is displaced through a step which $\equiv \frac{1}{3} c \mathrm{C}^{\prime}$, since

$$
c \mathrm{C}^{\prime}: \mathrm{BC}^{\prime}=\mathrm{CC}^{\prime}: \mathrm{DC}^{\prime}=\text { area } \mathrm{ABC}: \text { area } \mathrm{ABCDA} .
$$

Again if the triangle $\mathrm{AC}^{\prime} \mathrm{D}$ is replaced by the triangle $\mathrm{AD}^{\prime} \mathrm{D}$, its centre of gravity gets a further displacement which $\equiv \frac{1}{3} C^{\prime} D^{\prime}$ so that in replacing $A B C D A$ by $A^{\prime} D A$ the displacement of its centre of gravity is compounded of $\frac{1}{3}$ of $\mathrm{C}^{\prime} \mathrm{C}^{\prime}$ and $\frac{1}{3}$ of $\mathrm{C}^{\prime} \mathrm{D}^{\prime}$, i.e. is $\equiv{ }_{3}^{\prime} c \mathrm{D}^{\prime}$, and the consequent displacement of the centre of gravity of the whole polygonal area is $\equiv \frac{1}{3} d \mathrm{D}^{\prime}$, since

$$
d D^{\prime}: c D^{\prime}=D^{\prime}: D_{1}^{\prime} D^{\prime}=\text { area } A B C D A \text { : area } A B C D D_{1} C_{1} B_{1} A \text {. }
$$

Similarly in replacing the area $A B_{1} C_{1} D_{1} A$ by the area $A D_{1}{ }^{\prime} D_{1} A$, the centre of gravity of the whole has been displaced through $\frac{1}{3}$ of the step $d_{1} \mathrm{D}_{1}{ }^{\prime}$, so that we can reach the centre of gravity of the original area by constructing that of the triangle $A D^{\prime} D_{1}^{\prime}$ and making a step from that, which is $\frac{1}{3}$ of the step compounded of $\mathrm{D}^{\prime} d$ and $\mathrm{D}_{1}^{\prime} d_{1}$.

It follows that $\mathrm{G}$ is the centre of gravity of the given area.

It may be remarked that this method of construction is applicable to any plane area, whether forming a single polygon, or several, if it is bounded by straight lines, and whether the polygons are convex or not. It even applies to areas bounded by autotomic rectilinear circuits, if we keep to the usual conventions as to the sign and "multiplicity" of the various compartments, as explained by De Morgan ("Extension of the Word Area," Camb. and Dub. Math. Journal, Vol. 5 (1850), p 139), and earlier by A. L. F. Meister ("De Genesi et Indole Figurarum Planarum," Nov. Com. Gött, Tom. I. (1769-70), p. 144).

In the case of an area bounded by several separate polygonal circuits, it is only necessary to introduce double straight lines leading from a point in the perimeter of one disconnected part to a point in the perimeter of another, and back, and to continue this process till all the separate parts are joined up so as to form an area bounded by a single rectilinear circuit which includes each of these double lines, traversed once in one sense and once in the opposite sense. 
For example, in Fig. 2 the two areas $\mathrm{ABCD}$ and EFG being given, the single circuit ABCDEFGEDA bounds the whole area, the connecting line $\mathrm{DE}$ being traversed in both directions.

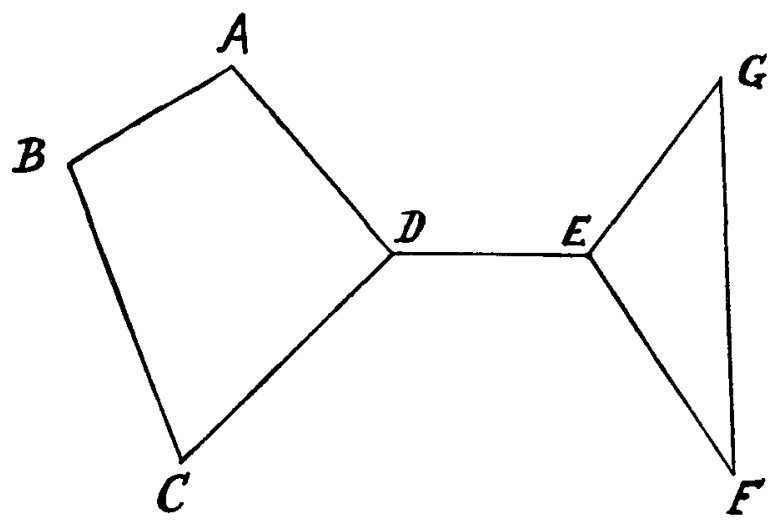

Fig. 2.

The method of construction as applied to the general case of the area enclosed by a single circuit $\mathrm{AB}_{1} \mathrm{~B}_{2} \ldots \mathrm{B}_{m} \mathrm{C}_{n} \mathrm{C}_{n-1} \mathrm{C}_{n-2} \ldots \mathrm{C}_{2} \mathrm{C}_{1} \mathrm{~A}$ may be stated thus:-

Let $\mathrm{B}_{1} \mathrm{~B}_{2} \quad$ il to $\mathrm{AB}_{2}$ meet $\mathrm{B}_{2} \mathrm{~B}_{3} \quad$ in $\mathrm{B}_{2}{ }^{\prime}$.

$$
\begin{aligned}
& \text { " } \mathrm{B}_{r}{ }^{\prime} \mathrm{B}_{r+1}^{\prime},, \mathrm{AB}_{r+1}, \quad \mathrm{~B}_{r+1} \mathrm{~B}_{r+2}, \mathrm{~B}_{r+1}^{\prime} . \\
& \text { " } \mathrm{B}_{m-1}^{\prime} \mathrm{B}_{m}{ }^{\prime},, \mathrm{AB}_{m} \quad, \quad \mathrm{~B}_{m} \mathrm{C}_{n} \quad \text { "B } \mathrm{B}_{m}^{\prime}
\end{aligned}
$$

followed by a similar construction, interchanging $C$ 's and B's, $m$ and $n$. Then

$$
\begin{aligned}
& \text { Let } \mathrm{B}_{2} b_{2} \| \text { to } \mathrm{B}_{1} \mathrm{~B}_{3} \text { meet } \mathrm{B}_{1} \mathrm{~B}_{2}^{\prime} \text { in } b_{2} \text {. }
\end{aligned}
$$

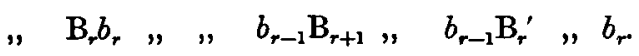

$$
\begin{aligned}
& " \quad \mathrm{~B}_{m} b_{m}, \quad, \quad b_{m-1} \mathrm{C}_{n}{ }^{\prime},, \quad b_{m-1} \mathrm{~B}_{m}^{\prime}, b_{m}
\end{aligned}
$$

with a similar construction, interchanging $\mathrm{C}$ 's and $\mathrm{B}$ 's, $m$ and $n$.

Let $\quad \mathrm{B}_{m}{ }^{\prime} \mathrm{O} \equiv \mathrm{OC}_{n}{ }^{\prime}, \quad \mathrm{AO} \equiv \mathrm{OA}^{\prime}, \quad \mathrm{A}^{\prime} b_{m}{ }^{\prime} \equiv \mathrm{B}_{m}{ }^{\prime} b_{m}, \quad b_{m}{ }^{\prime} c_{n}{ }^{\prime} \equiv \mathrm{C}_{n}{ }^{\prime} c_{n}$, $A G \equiv \frac{1}{3} A C_{n}^{\prime}$.

Then $\mathrm{G}$ is the required centre of gravity. 


\section{Centre of Gravity of a System of Particles.}

Let $n$ particles $m_{1}, m_{2}, \ldots m_{n}$ be situated at points $A_{1}, A_{2}, \ldots A_{n}$; and let $\mathbf{A}_{12}$ be the centre of gravity of $m_{1}$ and $m_{2}, A_{23}$ that of $m_{2}$ and $m_{3}$, and, generally, let $A_{r s}$ be that of $m_{r}$ and $m_{r}$.

Let the straight lines $\mathbf{A}_{1} \mathbf{A}_{2}$ and $\mathbf{A}_{12} \mathbf{A}_{3}$ intersect in $\mathbf{A}_{123}$.

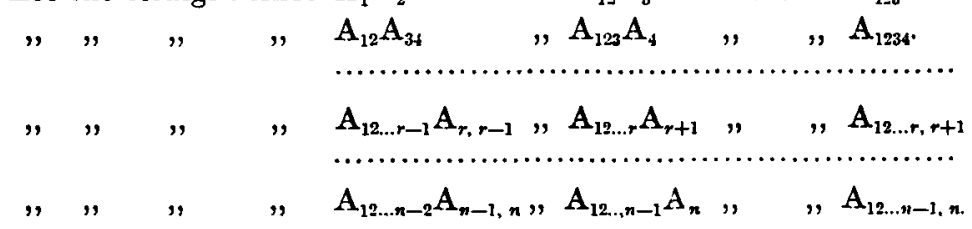

Then $A_{12 \ldots n-1, n}$ is the centre of gravity of the system. The reason is obvious.

This construction fails when the particles are all in a straight line. In this case we may introduce a new particle $m_{0}$ at $A_{0}$, a point not in line with the rest, and having constructed the point $A_{012 \ldots n}$ by the above method, draw $A_{0} A_{012 \ldots n}$ to meet the line of the given system in $G$, which will be the centre of gravity of the system $m_{1}, m_{2}, \ldots m_{n}$.

\section{Polygonal Plane Areas (Second Method.)}

It will suffice to illustrate this method, which is based on II. by a single example. (See Figure 3 ).

Let $\mathrm{AB}_{1} \mathrm{~B}_{2} \mathrm{~B}_{3} \mathrm{~B}_{4} \mathrm{~B}_{5} \mathrm{~A}$ be a plane polygon, and let $\beta_{1}, \beta_{2}, \beta_{3}, \beta_{4}$ be the mid points of $B_{1} B_{2}, B_{2} B_{3}, B_{3} B_{4}$ and $B_{4} B_{5}$ respectively.

Let $\beta_{1} \beta_{2}$ meet $\mathrm{AB}_{2}$ in $\mathrm{C}_{2}$ and let $\beta_{1} \beta_{12} \equiv \mathrm{C}_{2} \beta_{2}$.

" $\beta_{2} \beta_{3} \quad, \quad \mathrm{AB}_{3}$ " $\mathrm{C}_{3}, \quad, \beta_{2} \beta_{23} \equiv \mathrm{C}_{3} \beta_{3}$.

" $\beta_{3} \beta_{4} \quad, \quad \mathbf{A B}_{4}, \mathbf{C}_{4} \quad, \quad, \beta_{3} \beta_{34} \equiv \mathrm{C}_{4} \beta_{4}$.

Let $\beta_{1} \beta_{23}$ meet $\beta_{12} \beta_{3}$ in $\beta_{123}$.

" $\beta_{12} \beta_{34}, \quad \beta_{123} \beta_{4}, \beta_{1334}$.

Let $A G \equiv \frac{2}{3} A \beta_{1334^{*}}$. Then $G$ is the centroid.

To prove this result, we note the steps from $A$ to the centres of gravity of the triangles $A_{1} B_{2}$ and $A_{3} B_{3}$ are respectively $\frac{2}{3}$ of $\mathrm{A} \beta_{1}$ and $\frac{2}{3}$ of $A \beta_{3}$, so that the step from $A$ to the centre of gravity of the figure $\mathrm{AB}_{1} \mathrm{~B}_{2} \mathrm{~B}_{3} \mathrm{~A}$ will be $\frac{2}{3} \mathrm{~A} \beta_{12}$. Similarly the steps from $A$ to the centres of gravity of the figures $A B_{2} B_{3} B_{4} A$ and $\mathrm{AB}_{3} \mathrm{~B}_{4} \mathrm{~B}_{5} \mathrm{~A}$ will be respectively $\frac{2}{3}$ of $\mathrm{A} \beta_{23}$ and $\frac{2}{3}$ of $\mathrm{A} \beta_{3 \mu}$. Hence by II. $G$ is the centroid of the area of the polygon $A B_{1} B_{2} B_{3} B_{4} B_{6} A$. 
This construction can be modified in the following manner:Take $A \gamma_{1} \equiv \frac{2}{3} \mathbf{A} \beta_{1}, \mathbf{A} \gamma_{2} \equiv \frac{2}{3} \mathbf{A} \beta_{2}$, etc., and in the rest of the construction substitute $\gamma_{1}, \gamma_{2} \ldots \gamma_{12}$, etc., for $\beta_{1}, \beta_{2}, \ldots \beta_{12}$, etc. Then $\gamma_{1234}$ is the required centre of gravity. With this modification it is unnecessary, in dissecting the polygon into triangles, to draw all the dissecting lines from the same vertex A. Any dissection of the polygon into triangles will serve, provided the triangles form a succession such that every pair of adjacent triangles in the succession have one side in common.

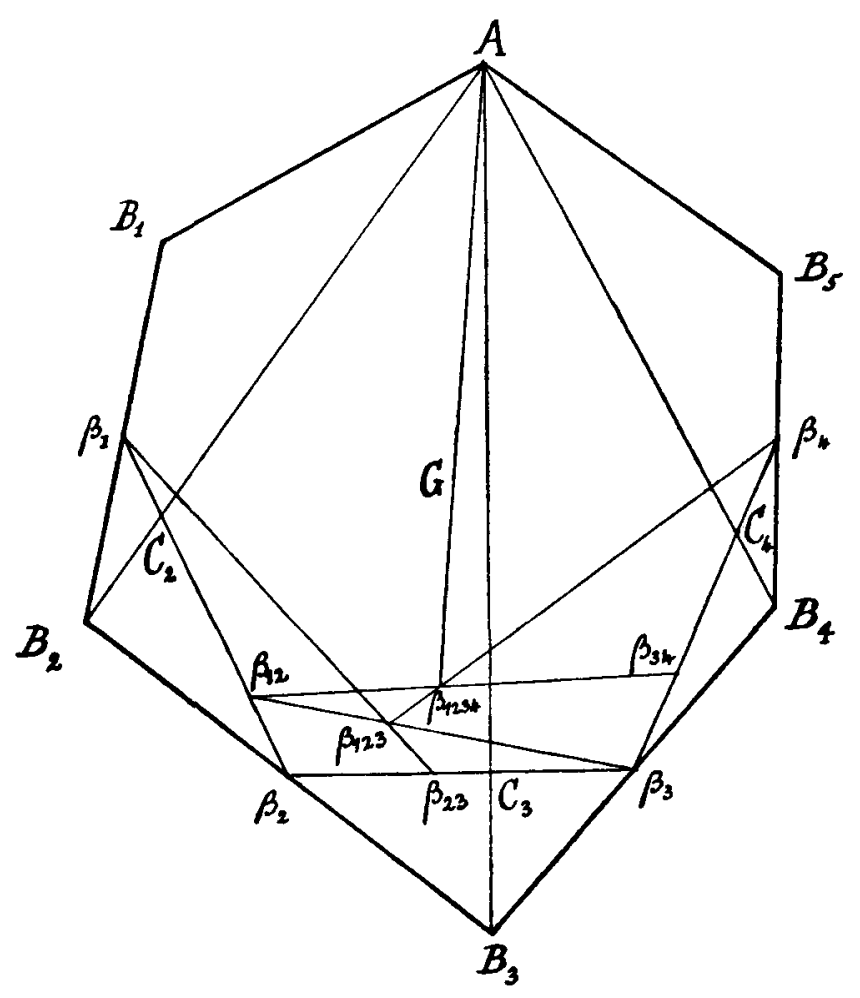

Fig. 3.

This method is, geometrographically considered, simpler than that of Section $I$., but it is to be noted that if set squares are used there is no need to draw the lines shown dotted in Fig. 1. 


\section{Additional Remarks.}

The method of II. is not confined to plane systems. And it is to be noted that we can apply this method to construct the centre of gravity of any system of bodies once we have obtained their centres of gravity and the centres of gravity of adjacent pairs when they are arranged in a succession or series, in any order.

For example to find the centre of gravity of a polyhedron, first suppose it divided by plane interfaces into tetrahedrons, and let these have an order in succession such that any two successive tetrahedrons have one face in common. It is then easy to construct the centre of gravity of each tetrahedron and of each pair of adjacent tetrahedrons in the succession. The problem is then reduced to that of II.

Section II. suggests a rather extended application of statical reasoning to prove geometrical propositions, analogous to the statical proof of the concurrence of the medians of a triangle.

If we have a set of points $(1),(2),(3) \ldots(n)(1,2),(1,3) \ldots(1,2,3)$, etc., which are situated so that the following sets of three are each collinear triads, viz.,

$$
\begin{array}{ll}
(1),(1,2),(2): & (2),(2,3),(3) ; \ldots \ldots(n-1),(n-1, n),(n) ; \\
(1),(1,2,3)(2,3) ; & (1,2)(1,2,3),(3) ; \text { etc. }
\end{array}
$$$$
r \ldots \ldots(1, \ldots r-1),(1, \ldots r),(r) ;(1, \ldots r-2),(1, \ldots r)(r-1, r) ; \ldots \ldots
$$

[where $(1 \ldots r)$ is a contraction for $(1,2,3 \ldots r)$ ]

Then a similar set of collinearities will hold good for the system of points when the fundamental set (1), (2)...(n) are taken in any other order of succession, and the points $(a, b, c \ldots k)$, $(p, q, \ldots t)$ and $(a, b, c \ldots k, p, q \ldots t)$ will be collinear when $a, b, c \ldots k, p, q, \ldots t$ are any of the numbers $1,2, \ldots n$.

Thus when $n=3$ we can choose arbitrarily the point $(1,2,3)$, and the remaining points $(1,2),(1,3),(2,3)$ are thereby determined by intersections of pairs of lines, or we can choose, say, the points $(1,2),(1,3)$ arbitrarily on the lines joining (1) and (2), (1) and (3), after which the two remaining points $(2,3)$ and $(1,2,3)$ are determined.

When $n=4$ we may choose arbitrarily on certain lines the three points $(1,2),(2,3),(3,4)$, after which the remaining derived points $(1,3),(1,4),(2,4),(1,2,3),(2,3,4),(3,4,1),(1,2,4),(1,2,3,4)$ 
are determined by intersections of pairs of lines, giving altogether 19 sets of 3 collinear points : but in this case there are 6 triads of points which are collinear though the lines have not been drawn. Thus there are six collinearity theorems for such a set of points.

In the general case where $n$ has any positive integral value, the total number of the principal and derived points is $2^{n}-1$. Amongst these are $\frac{1}{2}\left(3^{n}+1\right)-2^{n}$ triads of collinear points. Of these collinearities, $2^{n+1}-3 n-1$ are given in the construction of the derived points, leaving $\frac{1}{2}\left(3^{n}+3\right)-3.2^{n}+3 n$ as the number of triads, the assertion of the collinearity of each of which is a theorem. Thus for $n=3$ there are no theorems of this sort; for $n=4$ there are six theorems; for $n=5$ there are forty-two theorems, and so on.

The dually correlative theory, in which concurrent lines take the place of collinear points, and vice versa, will obviously be true also. It is associated with a method of constructing the line of action of the resultant of any number of coplanar forces acting along lines (1), (2), (3), ..( $n)$, when the lines of action $(1,2)$, $(2,3) . .(n-1, n)$ of the resultants of successive pairs have been determined. The line $(1,2,3)$ is got by joining the intersections of $(1,2)$ with $(3)$ and of $(1)$ with $(2,3)$; then the line $(1,2,3,4)$ by joining the intersections of $(1,2,3)$ with $(4)$ and of $(1,2)$ with $(3,4)$, and so on, until we get the line $(1,2,3 \ldots n)$, which will be the line of action of the resultant.

This theory and method, however, is not applicable in general to the case of non-coplanar forces, but only if the lines whose mutual intersections should determine points in the figure do really intersect one another.

The construction of the line $(1,2,3 \ldots n)$ also fails in the case of coplanar forces if the forces are all parallel. But in such a case we can introduce a new force $(0)$ and construct the line $(0,1,2, \ldots n)$; then the line of action $(1,2, \ldots n)$ of the original set will be parallel to them and will pass through the intersection of $(0,1,2 \ldots n)$ with the line $(0)$. By a similar expedient we can evade the difficulty arising from the parallelism of any two lines whose intersection is required. 